\title{
Author Correction: CKIP-1 limits foam cell formation and inhibits atherosclerosis by promoting degradation of Oct-1 by REG $\gamma$
}

Jiao Fan, Lifeng Liu, Qingyan Liu, Yu Cui, Binwei Yao, Minghua Zhang, Yabing Gao, Yesheng Fu, Hongmiao Dai, Jingkun Pan, Ya Qiu, Cui Hua Liu (D), Fuchu He, Yu Wang \& Lingqiang Zhang

Correction to: Nature Communications https://doi.org/10.1038/s41467-018-07895-3, published online 25 January 2019.

The original version of the Supplementary information associated with this Article contained an error in Supplementary Fig. 1a, in which the blots for $\beta$-actin in the left panel and the blots for CKIP- 1 and $\beta$-actin in the right panel were inadvertently replaced with incorrect blots. The HTML has been updated to include a corrected version of the Supplementary information.

Published online: 22 September 2021

\section{Additional information}

Supplementary information The online version contains supplementary material available at https://doi.org/10.1038/s41467-021-25986-6. 